\title{
IMAGE FUSION BASED LUNG NODULE DETECTION USING STRUCTURAL SIMILARITY AND MAX RULE
}

\author{
Mohana Priya R, \\ Department of Electronics and Communication Engineering, \\ Sri Chandrasekharendra Saraswathi Viswa Mahavidyalaya (Deemed to be \\ University), \\ Kanchipuram, TamilNadu, India \\ mohanapriyadhinakar@gmail.com \\ Dr. Venkatesan P, \\ Department of Electronics and Communication Engineering, \\ Sri Chandrasekharendra Saraswathi Viswa Mahavidyalaya (Deemed to be \\ University), \\ Kanchipuram, TamilNadu, India \\ pv.ecekanchi@gmail.com
}

\begin{abstract}
The uncontrollable cells in the lungs are the main cause of lung cancer that reduces the ability to breathe. In this study, fusion of Computed Tomography (CT) lung image and Positron Emission Tomography (PET) lung image using their structural similarity is presented. The fused image has more information compared to individual CT and PET lung images which helps radiologists to make decision quickly. Initially, the $\mathrm{CT}$ and $\mathrm{PET}$ images are divided into blocks of predefined size in an overlapping manner. The structural similarity between each block of CT and PET are computed for fusion. Image fusion is performed using a combination of structural similarity and MAX rule. If the structural similarity between CT and PET block is greater than a particular threshold, the MAX rule is applied; otherwise the pixel intensities in CT image are used. A simple thresholding approach is employed to detect the lung nodule from the fused image. The qualitative analyses show that the fusion approach provides more information with accurate detection of lung nodules.
\end{abstract}

Keywords: CT/PET lung image, image fusion, structural similarity, fusion rules.

\section{INTRODUCTION}

Lung cancer detection in CT and PET images using statistical texture features is discussed in [1]. At first, the preprocessing of CT and PET lung images is made by contrast limited adaptive histogram equalization technique. Then Region Of Interest (ROI) is extracted. The features are extracted by Gray Level Cooccurrence Matrix (GLCM) and texture features. Fuzzy clustering means algorithm is used for classification of CT and PET lung images. Lung cancer diagnosis in CT and PET images using multiple image features and Support Vector Machine (SVM) is discussed in [2]. Features like entropy, mean gray level intensity and uniformity features are extracted from the ROI. The lung cancer classification is made by SVM.

Lung tumor classification using Multi Layer Perceptron (MLP) is described in [3]. At first, the lung nodules are segmented by thresholding method and $k$ means clustering. Then, GLCM and texture feature such as homogeneity, dissimilarity, angular second moment, standard deviation and mean are extracted. The classification is made by MLP and $k$-Nearest Neighbor $(k N N)$. CT lung image 
classification using feature extraction is discussed in [4]. The regular and irregular margins features are extracted and then segmentation is made by Otsu thresholding method. Features like compactness, solidity, convexity and circularity are extracted from the segmented nodules. MLP is used for the classification of lung mages.

Deep transfer learning based lung nodule classification in CT images is discussed in [5]. Initially, the input CT images are given to different convolution neural network based on Visual Geometric Group (VGG) for feature extraction. The classification is made by SVM, MLP, Random Forest (RF) and $k N N$ classifier. Lung nodule segmentation and classification based on SVM using CT lung images is discussed in [6]. At first, the input lung images are preprocessed by using several masks. The segmentation of CT lung images is made by thresholding and morphological operations. Features are extracted only from the ROI by using Hounsfield units. SVM is used for classification.

The classification and detection of lung cancer using deep learning is presented in [7]. The CT lung images are segmented by using U-Net architecture. The extracted features are classified by using VGG network. The classification of lung cancer stages using CT lung images using image processing techniques is presented in [8]. Initially, the input images are smoothed by median and Gabor filter. The image enhancement is also made. The image segmentation is made by watershed segmentation algorithm. Features like area, eccentricity and perimeter are extracted and the classification is made by SVM.

Lung cancer detection and classification based on image processing techniques is discussed in [9]. The input lung images are preprocessed by median filter to remove noise. The segmentation of lung image is made by watershed segmentation and high boost operator. The suspicious ROI is extracted and then the classification of lung nodule is made by SVM classifier. Pathological lung identification is discussed in [10] for classification and segmentation of lungs. The fuzzy connectedness algorithm is used for the segmentation of lung images. The $\mathrm{RF}$ classifier is used for classification.

The detection of lung cancer using CT lung image is discussed in [11]. The input CT lung images are preprocessed by cropping the image and median filter is applied to remove noise. The segmentation of lung images is made by masking method. The features are extracted by Haar wavelet transform, GLCM and Haralick features. The classification is made by artificial neural network. Fuzzy inference system based lung nodule classification and detection is described in [12]. After image enhancement, segmentation is made by active contour model and spatial features are extracted. The fuzzy inference system and SVM classifier is used for classification.

In this study, image fusion based lung nodule detection using CT and PET lung images is presented. The organization of the paper is as follows: Section 2 explains the methods and materials used in this study for CT/PET lung image fusion. The results and discussion about the fusion approach are explained in section 3. Section 4 concludes the image fusion approach for lung nodule detection using the combination of structural similarity and MAX rule.

\section{METHODS AND MATERIALS}

The CT/PET lung image fusion for lung nodule detection using the combination of structural similarity and MAX rule is shown in Fig. 1. AT first, the $\mathrm{CT}$ and PET lung images are divided into overlapping blocks of size 3x3. Each block in $\mathrm{CT}$ is compared with corresponding PET block by a structural similarity measure. Based on the computed measure, Max rule is employed for fusion. 
Int. J.Adv.Sig.Img.Sci, Vol. 5, No. 1, 2019

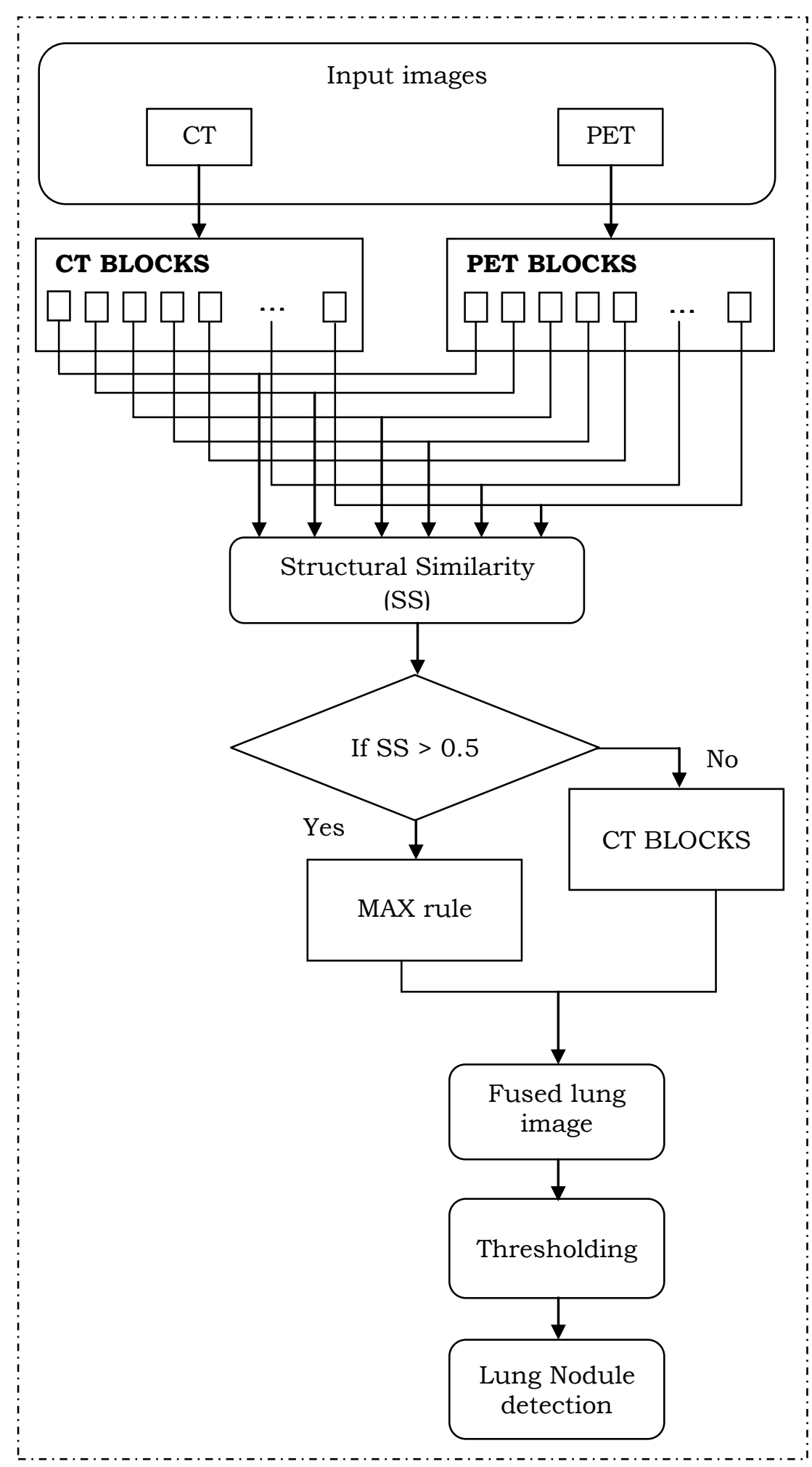

Fig. 1 Image Fusion based lung nodule detection 


\section{A. Structural Similarity Measure}

Structural similarity measure is used to find quality of image or videos in comparison with a reference. It shows the accurate error when compared to mean square error and peak signal to noise ratio. It is also used in video coding [13] and image de-noising [14]. It is used to change the structure of the image and gives the structural information.

The structural information gives the strong inter-dependency value for pixels which are spatially close. This measure is calculated by various windows in the image. Let us consider, the two windows $u$ and $v$ of same size $K x K$ is given by,

$$
\operatorname{Similarity}(u, v)=\frac{\left(2 \delta_{u} \delta_{v}+v_{1}\right)\left(2 \lambda_{u v}+v_{2}\right)}{\left(\delta_{u}^{2}+\delta_{v}^{2}+v_{1}\right)\left(\lambda_{u}^{2}+\lambda_{v}^{2}+v_{2}\right)}
$$

Where $\delta_{u}$ is the average of $u, \delta_{v}$ is the average of $v, \lambda_{u}^{2}$ is the variance of $u, \lambda_{v}^{2}$ is the variance of $v, \lambda_{u v}$ is the covariance of $u$ and $v$. It is based on the samples of $u$ and $v$ by the contrast (con), luminance (lumi) and structure (struc) is given by,

$$
\begin{aligned}
& \operatorname{con}(u, v)=\frac{2 \delta_{u} \delta_{v}+v_{2}}{\delta_{u}^{2}+\delta_{v}^{2}+v_{2}} \\
& \operatorname{lumi}(u, v)=\frac{2 \lambda_{u} \lambda_{v}+v_{1}}{\lambda_{u}^{2}+\lambda_{v}^{2}+v_{1}} \\
& \operatorname{struc}(u, v)=\frac{\delta_{u v}+v_{3}}{\delta_{u} \delta_{v}+v_{3}}
\end{aligned}
$$

where $v_{3}=v_{2} / 2$, the structural similarity with these three combinations is given by,

$$
\text { Similarity }(u, v)=\left[\operatorname{con}(u, v)^{A} \cdot \operatorname{lumi}(u, v)^{B} \cdot \operatorname{struc}(u, v)^{C}\right]
$$

The values of $A, B$ and $C$ are set to 1 . In this study, the input CT and PET lung images are divided into different blocks of predefined size, then these blocks are combined using the similarity measure obtained from (5) by MAX rule.

\section{B. MAX Rule Selection}

The MAXMIN rule is used in many computer vision applications such as data hiding approach [15] and video identification [16]. The function of MIN unit and MAX unit is to identify the minimum and maximum values of input data respectively. The MAXMIN rule for two class sub-problem is given in following equation.

$$
\begin{gathered}
R_{g, h}^{v}=\operatorname{MIN}\left(R_{g, h}^{v, 1}, R_{g, h}^{v, 2}, \ldots \ldots, R_{g, h}^{v, K u}\right) \\
R_{g, h}=\operatorname{MAX}\left(R_{g, h}^{1}, R_{g, h}^{2}, \ldots \ldots . ., M_{g, h}^{K_{g}}\right)
\end{gathered}
$$

where $v=1,2,3, \ldots ., n, K_{g}, g, h=1,2, \ldots . ., n g \neq h$ and the $R_{g, h}^{v, 1}=\left(v=1,2, \ldots . ., \quad K_{g}, g=1,2, \ldots ., K_{g}\right)$ is the output base. In this study, the MAX rule selection is employed for the fusion of CT and PET lung images. 


\section{RESULTS AND DISCUSSION}

The performance of image fusion of CT and PET images for lung nodule detection is discussed in this section. The system is evaluated using CT and PET lung images taken from the Reference Image Database to Evaluate therapy Response (RIDER) lung database [17-18]. Figure 2 shows the CT and PET lung images in the RIDER database.
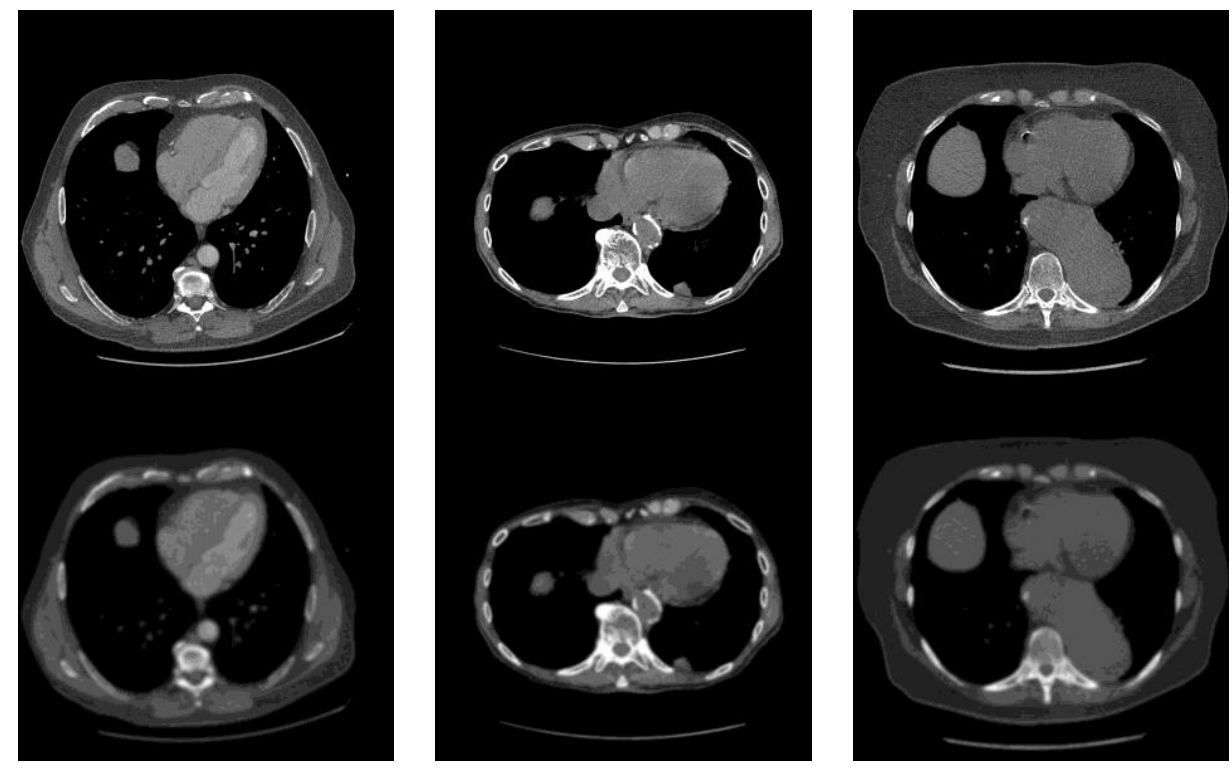

Fig. 2 CT (top row) and PET (middle row) lung images in RIDER database

At first, the CT and PET lung images are divided into non-overlapping blocks of size $3 \times 3$. Then the structural similarity between each block is computed using (5) and then MAX rule is applied using (7) for fusion if the structural similarity is greater than the 0.5. Figure 3 shows the fused lung image and lung nodule detected image using a simple thresholding approach.
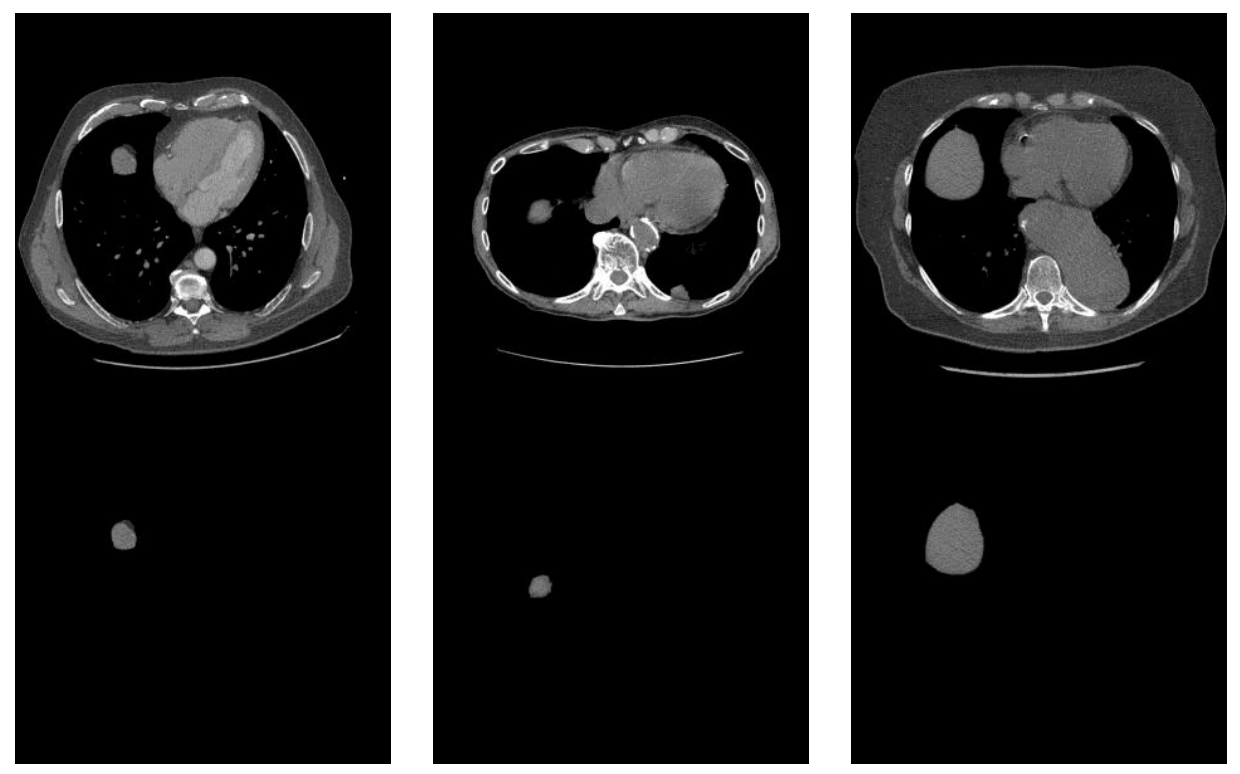

Fig. 3 Fused images (top row) and nodule detected images (bottom row) 
It is observed from the fused images and nodule detected images that the fusion approach provides better detection of lung nodules with exact boundaries of nodules in the images.

\section{CONCLUSION}

In this paper, fusion of $\mathrm{CT}$ and PET lung images for lung nodule detection is presented. It uses a combination of structural similarity and MAX rule for fusion in a $3 \times 3$ block of $\mathrm{CT}$ and PET lung images. At first, the structural similarity between the blocks of CT and PET is computed and MAX rule is applied based on the similarity measure. Results show that the lung nodules are detected accurately with the help of fused image. In future, the work can be extended to classify the lung nodules based on their abnormal severity. Also, the fusion can be performed in a frequency domain instead of fusing CT and PET images in the spatial domain.

\section{REFERENCES}

[1]. K. Punithavathy, M.M. Ramya, and S. Poobal, "Analysis of statistical texture features for automatic lung cancer detection in PET/CT images", International Conference on Robotics, Automation, Control and Embedded Systems, 2015, pp. 1-5.

[2]. N. Guo, R.F. Yen, G. El Fakhri, and Q. Li, "SVM based lung cancer diagnosis using multiple image features in PET/CT", IEEE Nuclear Science Symposium and Medical Imaging Conference, 2015, pp. 1-4.

[3]. S. Potghan, R. Rajamenakshi, and A. Bhise, "Multi-Layer Perceptron Based Lung Tumor Classification", International Conference on Electronics, Communication and Aerospace Technology, 2018, pp. 499-502.

[4]. Y.F. Riti, H.A. Nugroho, S. Wibirama, B. Windarta, and L. Choridah, "Feature extraction for lesion margin characteristic classification from CT Scan lungs image", International Conference on Information Technology, Information Systems and Electrical Engineering, 2016, pp. 54-58.

[5]. R.V.M. da Nóbrega, S.A. Peixoto, S.P.P. da Silva, and P.P. Rebouças Filho, "Lung Nodule Classification via Deep Transfer Learning in CT Lung Images", International Symposium on Computer-Based Medical Systems, 2018, pp. 244-249.

[6]. E. Rendon-Gonzalez, and V. Ponomaryov, "Automatic Lung nodule segmentation and classification in CT images based on SVM", International Kharkiv Symposium on Physics and Engineering of Microwaves, Millimeter and Submillimeter Waves, 2016, pp. 1-4.

[7]. R. Tekade, and K. Rajeswari, "Lung Cancer Detection and Classification Using Deep Learning", International Conference on Computing Communication Control and Automation, 2018, pp. 1-5.

[8]. A. Kulkarni, and A. Panditrao, "Classification of lung cancer stages on CT scan images using image processing", International Conference on Advanced Communications, Control and Computing Technologies, 2014, pp. $1384-1388$.

[9]. P.R. Katre, and A. Thakare, "Detection of lung cancer stages using image processing and data classification techniques", International Conference for Convergence in Technology, 2017, pp. 402-404. 
[10]. T. Revathi, and P. Geetha, "Lung segmentation and classification for pathological lung identification", International Conference on Computation of Power, Energy Information and Communication, 2016, pp. 148-153.

[11]. M. Vas, and A. Dessai, "Lung cancer detection system using lung CT image processing", International Conference on Computing, Communication, Control and Automation, 2017, pp. 1-5.

[12]. T.S. Roy, N. Sirohi, and A. Patle, "Classification of lung image and nodule detection using fuzzy inference system", International Conference on Computing, Communication \& Automation, 2015, pp. 1204-1207.

[13]. T. Zhao, J. Wang, Z. Wang, and C.W. Chen, "SSIM-based coarse-grain scalable video coding", IEEE Transactions on Broadcasting, Vol. 61, No. 2, 2015, pp. 210-221.

[14]. V. Bruni, D. Panella, and D. Vitulano, "Non local means image denoising using noise-adaptive SSIM", European Signal Processing Conference, 2015, pp. 2326-2330.

[15]. G.V. Moustakides, and V.S. Verykios, "A MaxMin approach for hiding frequent itemsets", Data and Knowledge Engineering, Vol. 65, No. 1, 2008, pp. $75-89$.

[16]. P.R. Futane, and R.V. Dharaskar, "Video gestures identification and recognition using fourier descriptor and general fuzzy minmax neural network for subset of indian sign language", International Conference on Hybrid Intelligent Systems, 2012, pp. 525-530.

[17]. K. Clark, B. Vendt, K. Smith, J. Freymann, J. Kirby, P. Koppel, S. Moore, S. Phillips, D, Maffitt, M, Pringle, and L, Tarbox, "The Cancer Imaging Archive (TCIA): maintaining and operating a public information repository", Journal of digital imaging, Vol. 26, No. 6, 2013, pp.1045-1057.

[18]. http://doi.org/10.7937/K9/TCIA.2015.OFIP7TVM 